\title{
Téoros
}

Revue de recherche en tourisme

\section{Le tourisme sportif d'eau vive. Développement de nouvelles territorialités}

\author{
Les cas de la France et de l'Afrique Australe
}

\section{Antoine Marsac}

Volume 28, numéro 2, 2009

Tourisme, sport et développement

URI : https://id.erudit.org/iderudit/1024803ar

DOI : https://doi.org/10.7202/1024803ar

Aller au sommaire du numéro

Éditeur(s)

Université du Québec à Montréal

ISSN

0712-8657 (imprimé)

1923-2705 (numérique)

Découvrir la revue

Citer cet article

Marsac, A. (2009). Le tourisme sportif d'eau vive. Développement de nouvelles territorialités : les cas de la France et de l'Afrique Australe. Téoros, 28(2), 21-28. https://doi.org/10.7202/1024803ar
Résumé de l'article

Cet article étudie l'émergence de nouveaux territoires de développement touristique. À partir d'une comparaison entre la France et l'Afrique, amorcée en 2005, cette enquête analyse les organisations du canoë et du rafting. Le tourisme d'eau vive est une forme d'excursion destinée à découvrir la nature, depuis 1932. Même si les activités originelles sont pratiquées en rivières, ces sports se déplacent des torrents aux espaces sauvages africains. L'étude historique du tourisme sportif d'eau vive montre que les canoéistes préfèrent les sites naturels aux espaces artificiels. Les espaces touristiques d'eau vive sont situés près des grandes villes. Le canoë demeure un sport de plein air même s’il se développe en milieu urbain. 


\title{
Le tourisme sportif d'eau vive : développement de nouvelles territorialités Les cas de la France et de l'Afrique Australe
}

\author{
Antoine MARSAC \\ Post-doctorant \\ Atelier SHERPAS \\ Université d'Artois \\ antoinemarsac@aol.com
}

\begin{abstract}
RÉSUMÉ: Cet article étudie l'émergence de nouveaux territoires de développement touristique. À partir d'une comparaison entre la France et l'Afrique, amorcée en 2005, cette enquête analyse les organisations du canoë et du rafting. Le tourisme d'eau vive est une forme d'excursion destinée à découvrir la nature, depuis 1932. Même si les activités originelles sont pratiquées en rivières, ces sports se déplacent des torrents aux espaces sauvages africains. L'étude historique du tourisme sportif d'eau vive montre que les canoéistes préfèrent les sites naturels aux espaces artificiels. Les espaces touristiques d'eau vive sont situés près des grandes villes. Le canoë demeure un sport de plein air même s'il se développe en milieu urbain.
\end{abstract}

Mots-clés: Tourisme d'eau vive, nouveaux territoires, nouveaux produits, globalisation.

Dans les sociétés contemporaines, la volonté de s'extraire de la ville souligne l'importance de retrouver un rythme qui a été dégradé par les conditions de vie urbaines. Les sports d'eau vive participent à ce mouvement de développement du tourisme, en adaptant le matériel aux cours d'eau propices à la navigation. Ces pratiques se définissent, à l'origine, comme des activités de découverte des espaces naturels. Elles regroupent trois activités, supports du tourisme (canoë-kayak, nage en eau vive et rafting). Ces activités sont considérées comme des pratiques sportives dans la mesure où elles nécessitent une certaine condition physique et requièrent des habiletés comme des réflexes spécifiques. En effet, lors d'une descente de torrent, le sportif doit éviter les rochers et les branches d'arbres au centre de l'eau. Dans un ouvrage, Bill Mattos (2003 : 113) définit l'eau vive comme «toute eau, généralement de rivière, qui comporte des rapides avec des zones d'écoulement fulgurant, où la forme du lit de la rivière provoque des vagues et des courants chaotiques et imprévisibles.». L'expression "eau vive», employée pour désigner une construction sociale, rassemble les sportifs adeptes de la rivière, de l'origine des pratiques jusqu'à celles d'aujourd'hui. Avec le développement du canoë, on peut s'interroger sur l'origine de cette expression. Les vocables «White water» aux États-Unis et «Wild water» au Royaume-
Uni désignent respectivement l'eau vive comme des eaux blanches ou sauvages. L'expression est une construction de la configuration occidentale des cours d'eau, qui opère une dichotomie «artificiel»/ «sauvage».

Pour appréhender l'organisation de ce tourisme sportif, nous nous intéresserons aux espaces naturels de pratique en eau vive. Les sportifs adeptes de canoë-kayak recherchent ces parties sauvages du réseau hydrographique. Alors que le monde dispose de rivières naturelles, des sites touristiques s'érigent en eau vive. Si l'activité s'enracine historiquement dans les rivières des pays occidentaux, elle ne s'y réduit pas. Des itinéraires touristiques ont été créés dans les pays africains. La notion «d'eau vive» regroupe des espaces naturels aménagés pour ce sport. Les kayakistes embarquent dans des espaces adaptés à la navigation de loisirs ou au tourisme. Ce sont donc les usages contemporains des embarcations qui détermineraient l'évolution des pratiquants dans l'espace. Maniant leur embarcation, ces kayakistes s'adaptent à l'environnement dans lequel ils naviguent (Mounet et Darolles, 2004; Pigeassou et Chaze, 2006). Cette étude interroge l'essor de nouveaux territoires de pratique. Les questions spatiales se posent : comment un site naturel peut-il servir d'infrastructure touristique et sportive durable? Le «tourisme sportif d'eau vive» constitue-t-il un vecteur de développement territorial? 
Le travail des sociologues Mao et Corneloup (2005) a mis en évidence le besoin de rechercher de nouvelles territorialités. Leur contribution porte sur les stratégies du tourisme d'eau vive dans les torrents tandis que leur étude géographique analyse l'activité autour de la mise en tourisme des Gorges de l'Ardèche. Mao et Corneloup (2006) se focalisent sur la prestation constituée par la location des embarcations dans les sites aménagés. Celle-ci prend en compte la consommation, précisant le rôle des pratiquants dans la conception de l'activité. Pierre Chazaud (2004) poursuit l'étude des sites d'eau vive à travers le management du tourisme de pleine nature. Des travaux spécifiques au tourisme se consacrent à la labellisation sectorielle d'espaces sportifs (Bouchet et Bouhaouala, 2007 : 65). À partir de modèles théoriques spécialisés (Bouchet et Kaach, 2004), il s'agit de montrer les enjeux et l'implication des sports d'eau vive dans l'essor d'infrastructures touristiques. Après un retour sur les conditions d'émergence du secteur, une comparaison sera opérée entre les territoires. Ce faisant, il convient de mesurer la portée de ce nouveau vecteur socio-économique, en interrogeant la transposition de ces pratiques sportives vers le tourisme en Afrique australe.

\section{L'émergence des territoires du tourisme d'eau vive}

La découverte des rivières par les premiers canoéistes citadins marque l'histoire de ce sport en étant à la fois ce qui fonde sa pratique dans son essence et l'usage par lequel cette pratique prend forme. La descente des cours d'eau s'impose en tant que distraction cultivée réservée à l'élite des canoéistes. L'étude des conditions d'apparition du tourisme analysée par Catherine Bertho-Lavenir (1999) prend la forme d'itinéraires, c'est-à-dire de territoires dédiés au voyage. À l'instar de cette auteure, limitons-nous à l'apparition des premières descentes de rivière en France. L'exemple de deux sites (alpestre et africain) illustre le développement de nouvelles territorialités. Le besoin d'explorer les torrents apparaît après la Première Guerre mondiale. L'excursionnisme préfigure l'arrivée du sport et du tourisme d'eau vive, à partir des années 1930. En fait, cette concomitance de l'essor du sport, du tourisme et de l'escapisme s'enracine dans un goût pour la découverte de nouveaux itinéraires. Ceux-ci sont utilisés pour le tourisme à la faveur de l'amélioration du matériel sportif (canoës, pagaies).

L'entre-deux-guerres apparaît comme une période propice à l'essor du tourisme de villégiature. Les rivières apparaissent véritablement comme des parcours d'eau vive à partir du moment où le tourisme d'eau vive se développe dans des lieux de plaisance. Puis, naviguer dans les torrents s'intensifie sous l'effet du besoin de s'évader de la ville. En effet, la frénésie du départ gagne peu à peu les canoéistes dans un engouement qui dépasse la simple promenade. En France, l'excursion en rivière s'impose comme modèle des pratiques avant le second conflit mondial. Un réseau constitué par le Touring Club de France (TCF) s'occupe dès 1932 d'aménager les parcours. Les lieux d'embarquement et de débarquement sont ainsi directement indiqués aux pratiquants par des guides touristiques spécialisés en canoë-kayak.

En 1937 se met en place un concours de construction d'embarcations spécifiques au tourisme dans le réseau hydrographique français. Les pagayeurs naviguent en rivière et s'inspirent également des guides, réalisés conjointement par ces sociétés nautiques constituées, autour de l'encouragement au tourisme. La «conquête» des torrents repose sur un

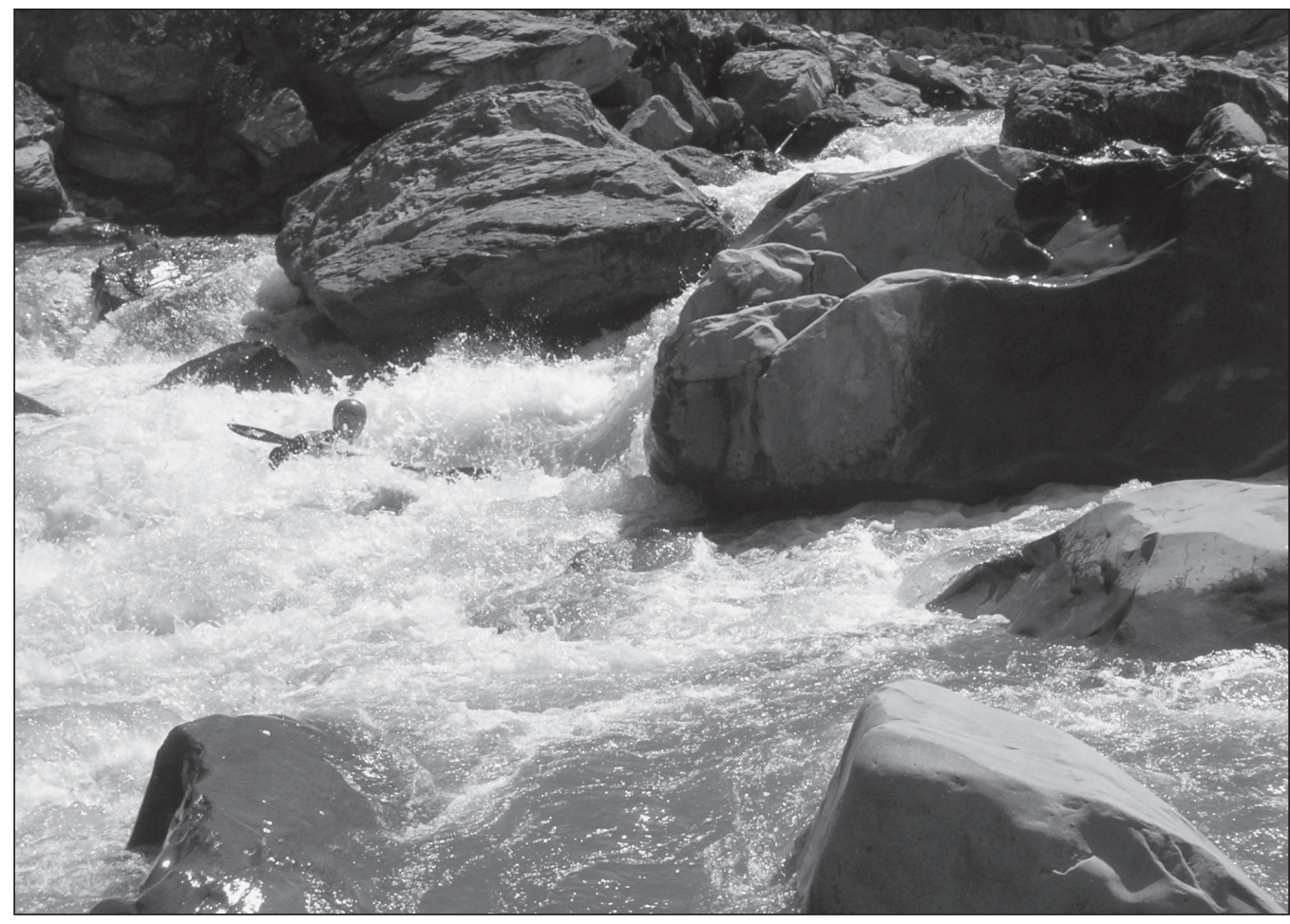

ILLUSTRATION 1 : Pagayeur dirigeant son embarcation dans un rapide (photo : Antoine Marsac). 
imaginaire canadien des grands espaces naturels, relayé par les médias. L'appréhension du milieu d'évolution constitue la principale qualité requise lors du séjour. Cette dimension d'exploration des territoires dans la pratique est souvent associée dans les mentalités à une activité de loisir. En effet, ce que le sens commun retient des activités du canoë-kayak, c'est l'expression «tourisme sportif». En fait, ce vocable rend compte de réalités fort disparates tant dans les pratiques que dans les parcours. En effet, celui-ci désigne à la fois la randonnée nautique sur les rivières de type Gorges de l'Ardèche, le kayak sportif dans les rivières en montagne ou les balades de quelques heures sur l'Ubaye, cours d'eau en crue au printemps, au moment où les torrents sont gonflés par la fonte des neiges. Toutes ces pratiques se différencient par l'intensité que sollicite l'activité (Lapierre, 1981, 1994). Il faut alors commencer par s'interroger sur l'origine de l'organisation des premiers pratiquants en eau vive pour saisir la relation qui lie les kayakistes citadins aux espaces de pratique.

Ce territoire sportif est marqué de pratiques, de valeurs, de symboles. Marc Augé (1992 : 56) parle de «surabondance spatiale». Sur la période contemporaine, le recours aux travaux spécialisés dans l'organisation du tourisme sportif s'avère nécessaire pour appréhender la dimension de l'organisation des pratiques d'eau vive en France. Postulant une similarité entre les sports dits «de montagne» (randonnée, escalade, canyoning) et ces activités nautiques, Pierre Chazaud (2004) est amené à opérer des rapprochements entre les pratiques de «glisse» et le tourisme sportif. Il se pose notamment la question de savoir comment un site sportif peut servir d'infrastructure touristique dans un territoire isolé.

Comme le réseau constitué par le Touring Club de France s'occupe d'aménager les parcours, le canoë-kayak reste une activité permettant une exploration des endroits reculés. En effet, la pratique en eau vive consiste à descendre ces portions de cours d'eau inaccessibles, insolites. Le contact avec la nature fonde la relation du citadin au milieu et reste une constante dans l'organisation des pratiquants. Comme toutes les activités de plein air, les sports d'eau vive ne conduisent pas uniquement à la compétition. Les pratiquants recherchent dans ces activités le contact avec la nature ou simplement cette découverte de sites méconnus, moins visités ou peu mis en valeur. Pour eux, les activités permettent un véritable loisir, une façon de réunir exploration, sport et défi ludique. La réussite en kayak tient au fondement sportif de la pratique (capacité à appréhender le sens de l'eau et à affiner le pagayage).

Le contact avec le milieu physique provoque, à la longue, une sensibilité des kayakistes aux éléments naturels. Celle-ci est utilisable pour constituer une pratique du kayak en activité sportive. Le couple kayakiste-pagaie se construit à travers une habileté motrice spécifique, le pagayage, gestuelle sportive. Il se prolonge, d'un côté par la solidarité entre le corps du kayakiste et son embarcation, de l'autre par la pénétration de la pagaie dans l'eau sur laquelle elle appuie. Cette relation au milieu par l'intermédiaire des sens modifie l'acteur - sa sensibilité, son information, sa capacité d'anticipation - tout en perturbant (modifiant) le milieu. L'équilibre du kayakiste conditionne les pratiques. C'est une qualité à acquérir dans l'embarcation avec l'anticipation des dangers du site. Il s'agit d'évoluer dans un territoire qui se transforme perpétuellement. Revenons maintenant sur la nature de ces changements spatiaux.

\section{Le tourisme à l'épreuve des transformations du territoire}

En France, l'aménagement destiné au tourisme d'eau vive est à dater précisément de 1933. En effet, une lettre est envoyée à Georges Bonnet, ministre français des Travaux publics de l'époque, afin de concilier eau vive et électrification. Elle mentionne l'édification de grands barrages destinés à satisfaire les besoins en électricité. Ces principes préfigurent les aménagements de rivières pour les besoins sportifs (de compétition). Mais le calendrier des rencontres sportives reste soumis aux aléas de l'hydrologie. Le tourisme sportif et les compétitions deviennent alors tributaires d'éléments extérieurs à la pratique : les décisions administratives et les lâchers d'eau des barrages. Des torrents alpestres sont recalibrés en de véritables gravières dans lesquelles naviguer perd de son intérêt.

Dans la plupart des cas, il n'y a plus assez d'eau pour manœuvrer l'embarcation. Les pagayeurs commencent à sélectionner des sites davantage propices, en termes de difficultés. En effet, pour les kayakistes, la richesse du milieu de pratique demeure soumise à la nature par la variation des courants (vague, rouleaux, remous) et les portions de rivières alpestres n'offrent plus assez de débits. Dès lors, l'activité s'organise selon la variation des niveaux d'eau et des indications consignées dans les guides. André Lapierre remarque que des compétitions sont déplacées ou annulées par manque d'eau. Les responsables s'emploient à faire coïncider leur calendrier avec les lâchers de barrages. Adeptes du tourisme dans une nature intacte, ils décrivent les premières réalisations comme des modifications majeures des espaces naturels (Lapierre, 1994 : 59).

La politique d'électrification, relancée au crépuscule de la Grande Guerre (par la loi de 1919 sur l'exploitation des cours d'eau du domaine français et d'outre-mer) fait tomber en désuétude ces itinéraires noyés par les retenus de barrages. La transmission de ce "patrimoine» de tourisme en canoë subit les affres d'un changement de population. Avec cent sept prisonniers de guerre et une bourgeoisie ascendante, quatre cents canoéistes des années trente arrêtent de pratiquer ce sport. Ces nombreux pionniers se tournent vers l'alpinisme ou la voile, sports en pleine expansion.

Les pratiques se prolongent par-delà les espaces naturels. Dès le début des années soixante, le canoë-kayak ne se pratique plus uniquement dans la nature, mais dans des parcours aménagés pour les besoins sportifs. Des sites sont érigés pour accueillir les compétitions et l'initiation des sportifs. Cette «pluralité spatiale» des modalités de pratique s'applique lorsque les kayakistes considèrent que leur espace de jeu est menacé. En 1974, le président d'honneur de la Fédération française canoë-kayak s'exprime dans ce sens :

«Nous essayons de créer, de soutenir une politique de défense du canoë-kayak d'eaux vives, menacé de mort (et avec elle une part de la vie que nous aimons), par les grands maux apparus depuis vingt ans à son égard, mais dont certains 
concernent un domaine beaucoup plus vaste : disparition des eaux libres, pollution des eaux restantes, effets néfastes de la loi de 1898 s'opposent à des besoins sociologiques plus récents.» (Bonnigal, $1974: 4$ )

Cet extrait montre comment la destruction de nombreux parcours naturels apparaît comme un fait marquant la pratique du canoë-kayak. Tous les parcours d'eau vive ne sont plus praticables. L'électrification anéantit les torrents comme les espaces touristiques. La pollution et les constructions de barrages raccourcissent le cours des rivières. Cela se répercute sur la pratique sportive dans la mesure où l'espace d'évolution des kayakistes se restreint.

Le contexte d'après-guerre a deux conséquences sur les pratiques touristiques en eau vive. D'une part, la vocation de ces descentes passe du tourisme excursionniste à l'hédonisme dans la nature. L'aspect sportif du canoë succède à l'exploration. Cette nouvelle forme de loisirs sportifs en montagne s'enracine dans la tradition des sports d'eau vive, nécessitant une bonne condition physique pour faire face aux difficultés de la rivière (rochers, masse d'eau) et contrôler son embarcation. D'autre part, l'émergence de nouvelles territorialités induit une adaptation des touristes aux courants des fleuves du monde entier. En témoignent les descentes de rivière en Afrique australe, sur le Zambèze en 1950, à la hauteur des chutes Victoria, côté zambien et sur le Nil blanc en Ouganda. À l'instar des travaux traitant de l'apparition de sites touristiques (Gagnon, 2007; Augustin et al., 2008), étudions maintenant ces nouvelles formes d'exploration en eau vive, en identifiant les territoires sur lesquels se réalisent les pratiques sportives.

\section{L'exploration de l'eau vive : un nouveau tourisme?}

Si les sports d'eau vive sont restés pendant longtemps l'apanage des sociétés occidentales, les espaces sur lesquels pratiquent les kayakistes français se sont déplacés vers les pays d'Afrique. Des centaines de pratiquants français sont partis vers ces contrées lointaines à la recherche d'une autre manière de pagayer. Sur place, ils ont trouvé les endroits propices à l'exploration (Nil, Zambèze, Gange, etc.) au contact des populations locales. Il s'agit d'une sorte de retour aux origines de l'activité, dans lequel chacun redécouvre les joies et la rusticité de la rivière : l'angoisse qui saisit le kayakiste au moment d'embarquer, la reconnaissance des difficultés aux abords des passages les plus délicats. Les guides locaux les encadrent dans les eaux tumultueuses de leur fleuve, mais l'activité est également empreinte des propriétés du monde occidental. Pour les clients, la pratique est choisie et non utilitaire, car son déroulement s'inscrit dans une temporalité calquée sur le modèle libéral de la prestation de service.

Le canoë-kayak, activité nautique et terrestre, renvoie à la dimension originelle du voyage des citadins. En effet, comme nous venons de le voir avec l'exemple des pagayeurs s'adonnant aux descentes touristiques de l'Ubaye, l'évasion de la ville occupe une place déterminante dans la formation d'un goût pour la nature et l'exploration de nouveaux territoires (Gammon et Robinson, 1997). Aussi, il s'avère important de revenir sur un autre contexte urbain exposant la relation qui lie des groupes de pratiquants à un cours d'eau.
L'urbanisation effrénée génère la formation d'un rapport social particulier des citadins aux espaces rivulaires. L'étude repose sur d'importantes interactions entre Africains et Français «expatriés». Ces groupes rencontrent des difficultés pour pratiquer le canoë-kayak dans un milieu naturel changeant. En effet, il s'agit de rapports doux à la nature tandis qu'il existe un rapport "dur» aux éléments. Pour illustrer ce cas, présentons trois types d'expériences de différents registres. La première est appelée "glisse dans l'eau ", tels ces rituels d'enterrement de vie de garçon dans lequel le pratiquant s'engage pleinement. La navigation en eau vive se réalise dans un milieu peu aménagé. L'apprentissage du pagayage dans son rapport à l'eau et à la glisse est conditionné par un contact à même le corps. Or, le kayak se limite à un déplacement et non à une immersion. Naviguer, c'est avant tout glisser sur l'eau, sans y pénétrer, avec le moins de résistance possible au milieu. La seconde expérience est la "glisse sur l'eau » à travers des cas où le pratiquant joue à filer et à tracer des courbes sur les surfaces anatomiques du clapot et les échines des courants en épousant les vagues et mouvements d'eau. Les kayakistes parisiens naviguant en Ubaye adoptent ce rapport sensible conditionné par l'influence du groupe sur le débutant. Ce pratiquant aspirant glisseur n'est pas un «sportif de l'extrême», mais un joueur, un «bricoleur», qui ajuste ses trajectoires au gré de sa perception de la nature et de dispositions en phase avec les attentes des responsables du groupe. Le cycle des saisons et les couleurs de l'eau conditionnent le type de pratique. Le développement de nouvelles territorialités s'accompagne d'une intensification des pratiques impliquant une découverte accrue de l'eau. Enfin, le troisième type d'expérience sous-tend un usage de l'embarcation adaptée aux descentes de rivière et au kayak, celle de la navigation embarquée où le rapport de glisse se fait par médiation du bateau voguant grâce à des instruments intermédiaires de prolongement du corps, optimisant la glisse et la gîte (la pagaie, etc.). Manœuvrer en eau vive, ou pagayer, c'est aussi glisser d'une autre manière, par médiation du coup de pagaie et de l'assise dans l'embarcation, sans que le corps soit en contact direct avec l'eau (ce seuil étant délicat). Cela correspond à ce qu'avance Jean Baudrillard (1968: 66) lorsqu'il écrit que «le gestuel de contrôle supplante le gestuel d'effort». Lorsqu'il est en rapport avec la «glisse» et non l'extrême, le contact du corps avec l'eau n'est-il pas expérience sociale faisant appel à la sensibilité commune des pratiquants? La technique peut-elle se départir des sensibilités de chacun? Ces questions soulèvent le problème des limites de la découverte de nouveaux sites touristiques.

\section{Les limites de la mobilité des sportifs sur un torrent alpestre}

L'attrait des kayakistes pour l'Ubaye, torrent sans barrage, se fonde sur les paysages des vallées préservées de l'industrialisation et du tourisme de masse. Le Conseil général des Alpes de Haute-Provence a signé un contrat pour le schéma départemental de tourisme qui définit ses axes stratégiques autour de trois pôles prioritaires : le tourisme actif, sportif et de pleine nature. Ce contrat de développement des filières touristiques sensibles doit aboutir sur un plan de gestion 
durable. Retirée des places fortes du tourisme provençal, à une centaine de kilomètres de la Côte d'Azur, la vallée de l'Ubaye «façonne» la vie des derniers montagnards qui ne vivent pas en ville. Une seule localité, Barcelonnette, présente un caractère urbain dans lequel s'agrègent les services. Cette ville regroupe plus du tiers des habitants de la vallée. Le tourisme est devenu le premier secteur économique de l'Ubaye depuis l'après-guerre. (AFIT, $2004: 89$ ).

Les élus de quatre communes environnantes (outre Barcelonnette, il y a le Lauzet-Ubaye, Méolans-Revel, Jausiers et La Condamine) ont donné une impulsion au tourisme en s'appuyant sur l'initiative des pratiquants des sports de nature. La fréquentation des stations de ski (Pra-Loup, Vars) ne représente que la moitié de la charge touristique totale de la vallée. Dans ce contexte, le canoë-kayak supplante la randonnée pédestre et les autres sports de nature (parapente, vélo tout terrain, rafting) tant en nombre de pratiquants qu'en infrastructures. Cette activité demeure l'une des plus anciennes de la vallée. Apparue à la fin des années trente, elle fait figure de pratique pionnière parmi les produits touristiques proposés. Depuis, la fréquentation de la rivière s'est stabilisée après une phase de croissance de 1970 à 1990 (Aubert, 2005).

En dépit d'un développement spécialisé dans quelques activités, le potentiel économique s'avère largement sousexploité, en partie à cause de la non-prise en compte de ces pratiques, dans les schémas d'aménagement des cours d'eau. En effet, l'usage à des fins sportives, demeure absent des projets de rivières. Dès lors, le processus de développement est ralenti dans sa phase de "mise en tourisme» par une politique hydraulique restreinte sur le plan écologique. Des réalisations d'envergure comme celles des barrages apparaissent. Alors que les niveaux d'eau sont peu favorables aux activités, paradoxalement, les sports d'eau vive sont un des rares attraits touristiques pouvant participer au rééquilibrage territorial entre un touriste mobile et des locaux prompts à s'établir durablement dans la vallée.

Georges Balandier (1985: 79) traite de la question de la mobilité à partir de deux types : l'«ancré» et le «nomade». L'usage éphémère explore de nouveaux espaces jusqu'alors réservés aux connaisseurs. Par l'évolution du matériel et l'ancrage dans de nouveaux territoires, les sports d'eau deviennent des activités communes. Les pratiques de randonnées longent la rivière. Les usagers de passage adoptent une pluralité de pratique, en des lieux éphémères. Ils côtoient les résidents permanents des villages traversés. Cependant, c'est au niveau de la connaissance des lieux qu'il est possible de différencier l'usager de passage du permanent. Le souci de transmission, de démonstration d'un savoir traditionnel de la vallée de l'Ubaye est conditionné par ce rapport population locale/ kayakistes. Le tourisme n'est pas en reste dans la mobilité de «retour à la nature» comme en témoigne la massification de la pratique dans les Gorges à la fin des années soixante puis son déclin au tournant des années 1990. Entre 1960 et 1970, l'estimation du nombre d'embarquements passe de 4000 en 1961 à 77000 en 1969 (Lapierre, $1994: 64$ ). Les conceptions de la nature sauvage apparaissent interdépendantes de l'offre touristique en eau vive.

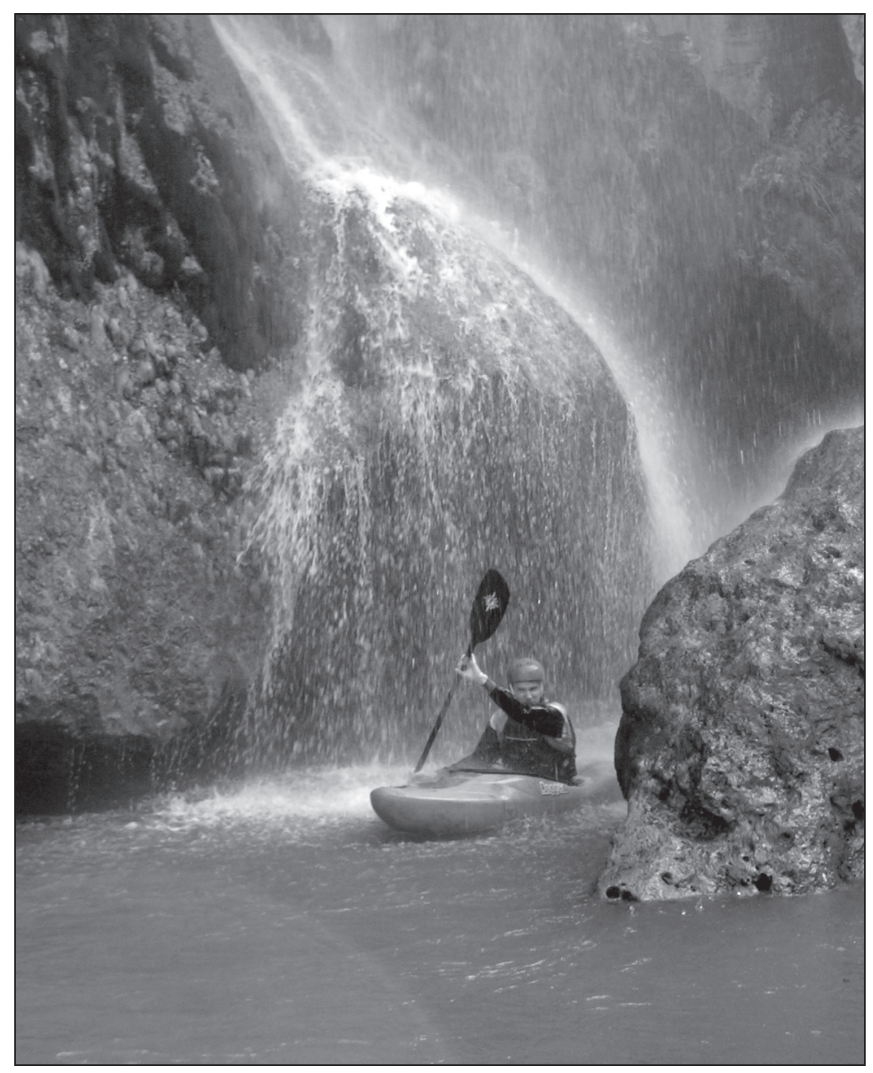

ILLUSTRATION 2 : Le canoë-kayak permet de découvrir, en aval d'une chute, des gorges abruptes (photo : Antoine Marsac).

\section{Le tourisme d'eau vive et la globalisation touristique}

En considérant l'eau vive dans sa dimension interculturelle et globale (MacDonald, 2006), cette partie vise à mettre en variation l'organisation des kayakistes lors des descentes de rivière, soumises aux contraintes d'un pays africain. Le Zambèze, en aval de Livingstone, est un haut lieu du kayak, un site de renommé mondiale depuis dix ans. Ce terrain sert de comparaison avec l'Ubaye, endroit jugé « authentique» dans sa naturalité pour les kayakistes. Ces derniers le considèrent comme un espace à contempler malgré les risques de crue. En eau vive, l'insécurité représentée par la nature est plus ou moins maîtrisée, la lenteur de déplacement est cependant toujours à l'ouvre. Elle s'est juste déplacée de la nature aux sites mondialisés. Le voyage constitue le fondement du «retour à la nature» bien que la mobilité s'accroît. Cette mobilité marquée par les véhicules remplace le symbole historique du campement " originel» et des croisières en Ubaye. Comme ce territoire n'a pas connu le chemin de fer, les canoéistes devaient soit arriver en voiture, soit charioter en portant l'embarcation. Les habitants de la vallée subissent les effets de la croissance des villes (rejet des citadins des métropoles, maintien de la «tradition» des travailleurs locaux).

Dans le contexte environnemental préservé de l'Ubaye, le canoë-kayak occupe une place de choix, car la fréquentation citadine demeure prégnante en été : «[L]es pratiques 
d'eau vive représentent une part prépondérante dans l'activité estivale (dix bases de raft et deux écoles de kayak) avec des pratiques sportives ou une découverte ludique[.]» (AFIT, $2004: 89$ ) En dépit de ces moyens de développement, le nombre de kayakistes n'aurait pas augmenté, selon les constats des moniteurs en Ubaye (Guides de la vallée de l'Ubaye, 14 octobre 2005). Ces guides qui travaillent sur cette rivière pointent même une désaffectation des sites au profit de destinations étrangères (Lapierre, 1994 : 78). Les kayakistes sont davantage enclins à partir dans des destinations lointaines. À ce titre, le Zambèze est devenu un «haut lieu » du tourisme sportif d'eau vive lorsque de nouveaux produits sportifs ont été proposés (saut à l'élastique), combinés aux safaris. En témoignent les «pack aventure», offre d'activités sportives proposée par les voyagistes, en France. Ces prestations sont couplées aux réservations d'hôtels de luxe ou de pavillons africains.

Désormais, un même territoire compte en son sein, des groupes usant différemment de l'espace en fonction de leurs normes et valeurs propres. L'appartenance est également remise en cause et chaque groupe tente de se légitimer par son ancrage au lieu. Le Lauzet, village de la vallée de l'Ubaye est investi par différentes populations (habitants de souche, néorésidents originaires ou non, résidents secondaires, touristes). Chacune possède un usage et une organisation propre de l'espace, tels les kayakistes originaires de la région parisienne. La cohabitation de ces différentes représentations sur le territoire des communes entraîne des conflits entre groupes, chacun se cachant derrière la conception floue de l'appartenance. De cette variété de groupe surgit immanquablement des usages, des enjeux et des stratégies territoriales divergentes. Les touristes habitués à l'eau vive de l'Ubaye quittent les lieux de villégiature qui leur étaient réservés en saison estivale pour investir des espaces naturels comme les fleuves africains. Parmi les acteurs qui fréquentent la rivière, il y a les clients des organisateurs de voyage. Ces touristes expriment le besoin de diversifier leurs pratiques (par les safaris en canoë en amont des chutes Victoria, par exemple). Dans ce cas, le territoire est approprié parce qu'il s'inscrit dans une dynamique propice à l'aventure par l'offre organisée.

L'identification de phases de développement (tableau 1) fait apparaître des disparités dans l'offre touristique. Le développement des territoires du tourisme sportif renvoie à l'organisation de l'industrie des loisirs à travers cette formation de l'exploitation commerciale. Des produits se développent (rafting, saut à l'élastique, etc.). Alors que le tourisme français apparaît segmenté, l'offre africaine se complète. La structuration des équipements dépend de ces pratiques légitimes et de l'utilité du sport pour le développement économique du pays. Les bases zambiennes sont un exemple d'exploitation sportive du site touristique mondialement connu des chutes Victoria. Elle sollicite les compétences de moniteurs sportifs.

\section{Des torrents à l'espace mondialisé}

Les sociétés occidentales produisent des formes de dépaysement à travers les produits d'aventure. Les kayakistes veulent se retrouver dans des mondes complètement différents. Le tourisme sportif y participe. Les clients ont un besoin de libération des affects poussé à l'extrême. Si les sociétés contemporaines fonctionnent sur les normes de retenu (la bienséance, le "politiquement correct»), elles ont moins tendance à ancrer l'individu dans un territoire. Pour compenser ce déficit d'enracinement, l'individu serait enclin à se tourner vers les grands espaces. De ce fait, les pratiquants des sports de nature s'engagent pleinement dans des sites où les éléments naturels abondent (Corneloup et al., 2001).

Cet ancrage sportif au territoire évolue, se forme, se déforme et n'est pas linéaire, immuable, continu. Il est fait de fractures, de discontinuités. L'ancrage dans une perspective temporelle est une construction dynamique. L'espace et le temps influencent l'individu et modifient son rapport à la nature, son territoire, ses représentations. Des formes de sensibilités à la nature différentes apparaissent chez les citadins. L'aspiration concrète mise en avant est celle d'une jouissance au présent d'une nature perçue comme abondante. Pour les kayakistes naviguant en Ubaye, la crue est, par exemple, synonyme d'optimisation des conditions de pratique. En revanche, les touristes en Afrique recherchent des conditions de sécurité comme en atteste la proximité de la ville. La globalisation remet en question cet attrait du non-contrôle des débits.

Si les sports dits «à risque » sont restés pendant longtemps l'apanage des sociétés occidentales, les espaces sur lesquels les touristes pratiquent se sont déplacés vers les pays d'Afrique (Zambie, Zimbabwe). Une centaine de pratiquants français sont partis vers ces contrées lointaines à la recherche d'autres manières de pagayer (Mattos, 2003 : 234). Sur place, ils ont trouvé les endroits qu'ils cherchaient depuis longtemps. En Afrique, sur le Zambèze, ce n'est pas la découverte de nouvelles cultures (celles des ethnies vivant près de la frontière

Tableau 1: Synthèse du développement de nouveaux territoires et de l'offre touristique

\begin{tabular}{c|c|c|c}
\hline & $\begin{array}{c}\text { Nouveauté territoriale } \\
\text { et offre touristique } \\
\text { (années 1930-1970) }\end{array}$ & $\begin{array}{c}\text { Exploitation du territoire : } \\
\text { infrastructures touristiques } \\
\text { (années 1980-1990) }\end{array}$ & $\begin{array}{c}\text { Développement de } \\
\text { produits touristiques } \\
\text { (années 2000) }\end{array}$ \\
\hline Ubaye (France) & Excursionnisme sur un torrent «sauvage» & $\begin{array}{c}\text { Développement de dix bases de rafting, kayak } \\
\text { et nage en eau vive }\end{array}$ & Diversification de l'offre sport/ tourisme \\
\hline Zambèze (Zambie) & $\begin{array}{c}\text { Spots (sites propices à la pratique) } \\
\text { et tourisme aux chutes Victoria }\end{array}$ & $\begin{array}{c}\text { Ouverture et gestion de huit compagnies } \\
\text { de rafting-safaris }\end{array}$ & Mélange safaris et raft, «pack aventure» \\
\hline
\end{tabular}

Source : Données compilées par l'auteur. 
entre la Zambie et le Zimbabwe à la hauteur des chutes Victoria) qui est en jeu, mais plutôt comment la passion du kayak cristallise les relations entre Français et Africains. Les deux groupes se retrouvent propulsés dans des interactions qui favorisent des débats sur les façons d'organiser l'activité, les manières de naviguer, l'utilisation du matériel. Mais, au final, c'est toujours la culture occidentale qui prime. C'est elle qui fixe ses cadres, y compris lorsque les guides locaux se sont approprié la pratique. Le kayak (originaire des cultures inuites) et le rafting demeurent des activités importées par des Occidentaux. Certes, l'activité canoë renvoie à l'imaginaire des grands espaces et provient de la naissance de populations aujourd'hui dominées par la culture occidentale (parfois qualifiée d'américaine). En termes de développement, les sports d'eau vive constituent une vitrine, générant des emplois spécifiques (Bouhaouala, 2008). Ce tourisme, pourvoyeur de postes, participe aux retombées économiques en Zambie, mais il cristallise un conflit entre locaux et touristes autour de l'eau.

Le conflit semble venir du fait que les territoires touristiques et les espaces de vie (village) ne sont pas juxtaposés, mais superposés selon des échelles différentes (Diénot et Theiller, 1999). Le sentiment d'appropriation génère des situations conflictuelles entre les groupes d'acteurs en présence sur le même espace. Plusieurs groupes possédant chacun des normes, des savoirs différents, parcourent l'espace du fleuve entraînant des droits de passage sur les pistes et les accès au fleuve Zambèze. L'ordre continental prend le pas sur l'organisation des pratiques urbaines qui ébranlent l'organisation de la descente des torrents.

\section{Conclusion}

Si le canoë-kayak peut être considéré comme un sport, mêlant effort et découverte, il constitue un moyen pour s'adonner au tourisme proche ou lointain. La pratique reste l'apanage des citadins cherchant à s'adonner à une activité physique dans un cadre naturel. L'activité prend essor dans le "retour à la nature» (Hervieu-Leger et Hervieu, 2005) comme en témoigne la massification de la pratique à la fin des années soixante. La tendance actuelle, au moins en France, porte à des descentes courtes sur des parcours choisis pour leur caractère sportif ou touristique. La croisière continue sur une rivière (Bertho-Lavenir, 1999) ne se pratique presque plus. L'ancrage spatial constitue un facteur décisif, car le territoire se modifie, d'autres lieux venant s'ajouter aux traditionnels torrents. L'inscription de groupes de kayakistes dans l'espace est à lier à la découverte de nouveaux territoires en interdépendance avec le défi sportif. L'exemple du Zambèze, fleuve d'Afrique australe illustre cette tendance à la découverte. Le territoire et le sentiment identitaire qui lui est associé semblent influencés par les touristes qui sont des milliers à visiter les chutes Victoria. Ils fondent leurs vacances sur le défi sportif (Viard, 2007 : 98). Le territoire actuel du tourisme d'eau vive est multiple, car il se base sur des lieux du passé et les nouveaux spots, sites propices à la pratique, sur les fleuves africains.

Mais, une question demeure en suspens. Elle concerne les orientations dues à la massification du tourisme sportif d'eau vive ainsi que leurs effets sur leurs espaces de pratique.
Les activités touristiques seront-elles placées sur le «même pied d'égalité » que le sport de compétition? Par le développement de ces sites artificiels, l'offre de pratique s'étend.

Si les sports d'eau vive constituent un moyen de découverte du territoire, les activités ne peuvent être vendues sans des produits annexes (safaris) qui complètent cette offre. L'association entre ces pratiques tend à masquer des conflits entre touristes et locaux. Pour toutes ces raisons, en France, les acteurs se rapprochent davantage des collectivités territoriales (communes et conseils généraux). En effet, ces assemblées délibérantes participent aux orientations en matière de développement territorial. Les politiques jouent un rôle décisif dans le développement d'activités. Outre le subventionnement des associations, elles donnent leur aval sur chaque projet d'aménagement de rivière et garantissent la «compatibilité» entre les sports d'eau vive et le tourisme sous toutes ses formes (Laplante, 1996). Il en va de la pérennisation des acteurs existants, ces enjeux dépassant largement le cadre sportif pour s'inscrire dans la structuration de l'offre touristique et le développement des territoires. Dans ces conditions, l'implantation de stades d'eau vive, espaces artificiels, s'érige comme un tournant décisif pour les pratiquants. Ces constructions ex nihilo sont destinées à compenser le manque d'activités occasionné par les problèmes de niveau d'eau. Le site solutionne en partie les problèmes d'accès aux rivières tout en déplaçant les enjeux vers l'exploitation des équipements, car le partage de l'eau s'opère entre sportifs et touristes.

\section{Bibliographie}

AFIT (2004) Guide de savoir-faire, Tourisme et loisirs de nature, développement touristique des territoires et sports de nature, Paris, AFIT. 248 p.

AUBERT, Jean-Pierre (2005) Président de l'Office de tourisme de Barcelonnette, communications personnelles.

AUGÉ, Marc (1992) Non-lieux, Introduction à une anthropologie de la surmodernité, Paris, Seuil. 150 p.

AUGUSTIN, Jean-Pierre (1995) Sport, géographie et aménagements, Paris, Nathan. 254 p.

AUGUSTIN, Jean-Pierre; Philippe BOURDEAU et Loïc RAVENEL (2008) Géographie des sports en France, Paris, Vuibert. 177 p.

BALANDIER, Georges (1974) [1985] Anthropo-logiques, Paris, Pocket. $280 \mathrm{p}$.

BAUDRILLARD, Jean (1968) Le système des objets, Paris, Gallimard. 288 p.

BERTHO-LAVENIR, Catherine (1999) La roue et le stylo. Comment sommes nous devenus touristes, Paris, Odile Jacob. 438 p.

BONNIGAL, Daniel (1974) «Promesses ou testament », La Rivière, nº 458, $4^{\text {e }}$ trimestre, p. 4-8.

BOUCHET, Patrick et Mohamed KAACH (2004) Afrique francophone et développement du sport: du mythe à la réalité, Paris, L'Harmatthan. $384 \mathrm{p}$.

BOUCHET, Patrick et Malek BOUHAOUALA (2007) «Les parcs d'activités sportives récréatives en forêt : diagnostics et perspectives durables ", Téoros, n² 26, p. 59-69.

BOUHAOUALA, Malek et Pierre CHIFFLET (2001) «Logique d'action des moniteurs des sports de nature : entre passion et profession ", STAPS, $\mathrm{n}^{\circ} 56$, p. 61-74.

BOUHAOUALA, Malek (2008) Management de la petite entreprise des loisirs sportifs. Une approche socioéconomique, Bruxelles, De Boeck. 158 p. 
BOURDEAU, Philippe (2007) «Les sports de nature comme médiateurs de l'entre-deux ville-montagne : vers un post-tourisme?», dans Lilli Monteventi-Weber, Chantal Descheneaux et Michèle Tranda-Pitton (dir.), Campagne-ville, le pas de deux. Enjeux et opportunités des recompositions territoriales, Lausanne, Presses polytechniques et universitaires romandes, p. 27-36.

BOYER, Marc (1996) Histoire de l'invention du tourisme. XVI'-XIX'e siècles. Origine et développement du tourisme dans le Sud-Est de la France, La Tour d'Aigues, Éditions de l'Aube. 332 p.

CHAZAUD, Pierre (2004) Management du tourisme et des loisirs sportifs de pleine nature, Voiron, Presses universitaires du sport. 345 p.

CORNELOUP, Jean; Malek BOUHAOUALA; Cécile VACHÉE et Bastien SOULÉ (2001) «Formes de développement et positionnement touristique des espaces sportifs de nature», Loisir et société, vol. $24, \mathrm{n}^{\circ} 1$, p. 21-46.

CORNELOUP, Jean (2005) «La place du marché dans le fonctionnement des loisirs sportifs de nature», Téoros, vol. 24, n 1, p. 55-62.

DAROLLES, Jean-Michel (1997) Canoë, eau vive, tourisme, Paris, AFIT. $349 \mathrm{p}$.

DIÉNOT, Josy et Didier THEILLER (1999) Les nouveaux loisirs sportifs en montagne, Les aventuriers du quotidien, Bordeaux, Éditions Maison des Sciences de l'Homme d'Aquitaine, $178 \mathrm{p}$.

EICHBERG, Henning; John BALE; C. PHILO et S. BROWELL (1998) Body Cultures: Essays on Sport, Space and Identity, New York, Routledge. $166 \mathrm{p}$.

GAGNON, Serge (2007) «Attractivité touristique et "sens" géo-anthropologique des territoires", Téoros, vol. 26, n 2, été 2007, p. 5-11.

GAMMON, Sean et Tom ROBINSON (1997) «Sport and Tourism : a conceptual framework", Journal of Sport Tourism, no 4, p. 10-17.

Guides de la vallée de l'Ubaye (2005) communication personnelles des moniteurs en Ubaye, entretien 14 octobre, Barcelonnette.

HERVIEU-LEGER, Danièle et Bertrand HERVIEU (2005) Le retour à la nature: «au fond de la forêt, ...l'État», Paris, Seuil. $234 \mathrm{p}$.

JAUBERT, J. (1933) «Grands barrages et Tourisme Nautique», La Rivière, $\mathrm{n}^{\circ} 251$, mai, p.114.

LAPIERRE, André (1981) Sports de pleine nature et pratiques sociales, analyse socioculturelle du canoë-kayak et de l'escalade, Mémoire cessionnaire pour le diplôme de l'INSEP, Paris.

LAPIERRE, André (1994) «Culture sportive française et culture créole», le canoë-kayak en France métropolitaine et à La Réunion, Université Paris XI-Orsay, DEASTAPS. 230 p.

LAPLANTE, M. (1996) L'expérience touristique contemporaine, fondements sociaux et culturels, Québec, Presses de l'Université du Québec. 189 p.

LATOUCHE, Serge (1989) L'occidentalisation du monde. Essai sur la signification, la portée et les limites de l'occidentalisation du monde, Paris, la découverte. $170 \mathrm{p}$.

MACDONALD, Kevin (2006) Global movements, action and culture, London, Blackwell. 251 p.

MATTOS, Bill (2003) Le canoë-kayak, Paris, La Martinière, Trad. Pierre Richard Rouillon. 256 p.

MAO, Pascal et Jean CORNELOUP (2005) «Approche géo-historique des formes de développement d'un territoire touristique et sportif de nature, La construction du haut lieu "Gorges de l'Ardèche" durant le XXe siècle», Loisir et société, vol. 28, n 1, Presses Universitaires du Québec, Sainte-Foy, p. 117-140.

MOUNET, Jean-Pierre et Paul CHIFFLET (1996) «Commercial supply for river water sports», International review for the sociology of sport, $\mathrm{n}^{\circ} 3$, R. Oldenbourg Verlag GmbH, Munich, p. 233-254.
MOUNET, Jean-Pierre et Jean-Michel DAROLLES (2004) «Le tourisme des sports d'eau vive, un exemple d'organisation des sports de nature entre local et national», dans C. Sobry (éd.), Le tourisme sportif, Paris, Presses universitaires du Septentrion. p. 287-295.

MOSS, Laurence (dir.) (2006) The Amenity Migrants: Seeking and Sustaining Mountains and their Cultures, Wallingford, Cabi Publishing. 336 p.

Office de tourisme de Barcelonnette (2005) Tourisme en vallée de l'Ubaye, entretien 14 octobre Barcelonnette.

PIGEASSOU, Charles et Jean-Pierre CHAZE (2006) «La rivière comme nouvel espace d'aventure : variation sur les usages et les enjeux de pratiques sportives de loisir et de tourisme», dans N. Bernard, Le nautisme. Acteurs, pratiques et territoires, Presses Universitaires de Rennes, p. 201214.

ROBERTSON, Roland (1992) Globalization. Social Theory and Global culture, Londres, Newsbury Park et New Delhi, Sage publications. 211 p.

TSHIKALA, K. Biaya et Gilles BIBEAU (1998) «Modernité indocile et pratiques subversives en Afrique contemporaine», Anthropologie et Sociétés, $\mathrm{n}^{\circ} 22$ (1), p. 5-13.

VIARD, Jean (2007) Penser les vacances, Paris, l'Aube. 232 p.

TÉOROS, vol. 28, nº 2, p. 21-28, ( 2009 